\title{
Topical Non-Pharmacological Treatment for Facial Seborrheic Dermatitis
}

\author{
Jaime Piquero-Casals (D) Doris Hexsel · Juan Francisco Mir-Bonafé • \\ Eduardo Rozas-Muñoz
}

Received: June 16, 2019 / Published online: August 8, 2019

(C) The Author(s) 2019

\begin{abstract}
Facial seborrheic dermatitis (FSD) is a chronic and relapsing inflammatory skin disorder occurring in areas of the face rich in sebaceous glands. It clinically manifests as erythematous scaly macules or plaques, often associated with pruritus. Although the pathogenesis of seborrheic dermatitis is not yet fully understood, Malassezia yeast, hormones, sebum levels, and immune response are known to play important roles. Additional factors including drugs, cold temperatures, and stress may exacerbate the condition. Currently, the available treatments do not cure the disease but relieve symptoms. Various pharmacological treatments are available, including antifungal agents, keratolytics, topical low-potency steroids, and calcineurin inhibitors. All of them provide several benefits, but they also have potential side effects. Seborrheic dermatitis tends to have a chronic,
\end{abstract}

Enhanced Digital Features To view enhanced digital features for this article go to https://doi.org/10.6084/ m9.figshare.9165284.

J. Piquero-Casals $(\bowtie) \cdot J$. F. Mir-Bonafé .

E. Rozas-Muñoz

Department of Dermatology, Hospital de la Santa Creu I Sant Pau, Barcelona, Spain

e-mail: jaime.piquero@e-campus.uab.cat

D. Hexsel

Brazilian Center for Studies in Dermatology, Porto

Alegre, RS, Brazil recurrent course. To avoid the long-term use of drugs, topical non-pharmacological products such as cosmetics or medical devices may improve clinical outcomes. Products with antimicrobial and anti-inflammatory ingredients such as zinc, piroctone olamine, dihydroavenanthramide, biosaccharide gum-2, and stearyl glycyrrhetinate may speed FSD recovery and avoid flare-ups. Finally, the use of specific cleansers, moisturizers, and sunscreens formulated as light creams or gel/creams should be strongly recommended to all FSD patients. We provide a brief review of the most used nonpharmacological cleansers, topical gel/creams, and specific sunscreens in the management of FSD.

Keywords: Cosmeceuticals; Cosmetics; Facial; Medical device; Seborrheic dermatitis; Topical treatment

\section{INTRODUCTION}

Facial seborrheic dermatitis (FSD), also known as facial seborrheic eczema, is a common chronic inflammatory skin disorder with a prevalence of up to $10 \%$ of the adult population [1]. Men are more likely to be affected than women, with a peak incidence in the third and fourth decades of life [2]. Clinically, it is characterized by itchy, erythematous, greasy, scaly 
plaques involving the forehead, eyebrows, glabella, and nasolabial folds [3]. In severe cases, crusting, oozing, fissures, and extension to other areas such as the cheeks may also occur [4]. Stress and winter weather aggravate the disease while summer weather and sun exposure usually improve it [5-7].

The pathogenesis of FSD is not yet fully established. Indirect evidence suggests the role of sebaceous glands and Malassezia yeasts in the disease [8]. Although the rate of sebum excretion is not increased in many patients with FSD, lesions are typically located in those areas that have the most and largest sebaceous glands [9]. A higher density of Malassezia yeast has been observed in some patients with FSD [10]. Malassezia are lipid-dependent yeasts that are able to exploit a narrow niche on sebum-rich skin [11]. It is thought that sebaceous secretions may facilitate the presence of Malassezia, which generate free fatty acids that then irritate the skin and induce dermatitis [9-11]. Not all patients with a high density of Malassezia develop the disease, so it may be possible that dysregulation of the immune system is required for their detrimental action [11]. Genetic studies have found up to 11 gene mutations and protein deficiencies linked to FSD or FSD-like phenotype in humans or mice, suggesting a genetic susceptibility [12].

A proposed scenario for developing FSD involves abnormal epidermal barrier (EPB) function due to genetic predisposition, and excessive or altered sebum composition that may exacerbate EPB disruption and provide an environment favorable to Malassezia growth. Disrupted EPB function allows Malassezia and its metabolites to enter and irritate the epidermis, eliciting an immune response from the host. This inflammatory response further disrupts epidermal differentiation and barrier function, and itching and scratching may cause further disruption: the result is a cycle of immune stimulation, abnormal epidermal differentiation, and barrier disruption [3-5].

There are three main pharmacological treatments for FSD: keratolytics, anti-inflammatories, and antifungal agents [13]. Keratolytics avoid scaling or crust formation and help improve the penetration of other topical treatments. Topical steroids and calcineurin inhibitors reduce inflammation, and antifungal agents decrease Malassezia populations. In addition, some agents such as ketoconazole have additional anti-inflammatory and antiandrogen properties [8]. Although the efficacy of these treatments has been demonstrated, the need for long-term treatment can cause side effects and cosmetic concerns that may limit their use. In these cases, the use of topical nonpharmacological therapies may be considered $[3,4,13]$ (Table 1$)$.

Corticosteroids are simple and effective but should be used sparingly because of their side effects-such as skin thinning and striaewhich may occur with long-term uninterrupted use over weeks or months. Creams or lotions containing the calcineurin inhibitors tacrolimus and pimecrolimus may be effective and cause fewer side effects than corticosteroids $[1,13]$, although they are more expensive. Such use is "off-label" as they are approved only for atopic dermatitis. Another reason they are not first-choice treatments is that the US Food and Drug Administration has concerns about a possible association with cancer [13].

Patients frequently have worries about the cosmetic symptoms of FSD, so reducing the

Table 1 Approaches to avoid the flare-up in FSD

Long-standing approaches

Educate (about gentle soaps, hydration, diet, reducing stress)

Hydrate (use daily light emollients, decrease steroid usage)

Use water-based emulsion sunscreen

Avoid excess makeup/medication

More recent approaches

Treat proactively; start drugs early for mild flare-ups and use for a short time

Reduce triggers for relapse

Use specific non-pharmacological treatments that act on multiple fronts 
clinical symptoms (erythema, scales, and itching) may help improve any psychological distress caused by the condition. Any concerns regarding the psychological impact of the disease should be highlighted through the use of the Dermatology Life Quality Index.

The aim of this review is to understand the management of FSD with non-pharmacological treatment, since it is a chronic disease that is difficult to treat. This article is based on previously conducted studies and does not include any studies with human participants or animals performed by any of the authors.

\section{Differences Between Topical Non- Pharmacological Treatments for FSD}

Non-pharmacological therapies include cosmetics, cosmeceuticals, and medical devices. Definitions are imprecise, meaning that regulations vary between countries. Some products are considered cosmetics or cosmeceuticals in one country, while in others they are considered medical devices [14].

Cosmetics are defined as substances or products used to improve the appearance of the skin without affecting the body's structure or function [15]. The Federal Food, Drug, and Cosmetic Act (FD\&C Act) defines cosmetics by their intended use, as "articles intended to be rubbed, poured, sprinkled, or sprayed on, introduced into, or otherwise applied to the human body for cleansing, beautifying, promoting attractiveness, or altering the appearance" [FD\&C Act, sec. 201(i)], the difference from a pharmaceutical drug being based on the intended use of the product.

The term "cosmeceutical" was introduced in 1961 by Raymond Reed, founding member of the US Society of Cosmetic Chemists, with reference to "active" and science-based cosmetics, and use of the term later became more widespread, thanks to the influence of Dr. Albert Kligman [16].

Medical devices include objects or substances used to help in the recognition, prevention, monitoring, treatment, and alleviation of diseases, and which achieve this "intended main effect" by physical rather than direct pharmacological, immunological, or metabolic means, although such effects may support the physical effects [14].

The correct use of this heterogeneous group of products can improve FSD treatment, enhancing the EPB function and avoiding excess use of topical drugs with potential side effects.

\section{Functional and Performance Cosmetic Ingredients in FSD}

Cosmetic products contain two types of ingredients: performance and functional [15]. Performance ingredients include all substances that cause changes in the appearance of the skin. The main performance ingredients used in FSD include piroctone olamine, stearyl glycyrrhetinate, avenanthramides, biosaccharide gum-2, and zinc salt of L-pyrrolidone carboxylate (zinc PCA), (Table 2). Functional

Table 2 Summary of useful ingredients for topical nonpharmacological FSD

\begin{tabular}{ll}
\hline Ingredient & Property \\
\hline $\begin{array}{l}\text { Piroctone olamine } \\
\text { Stearyl glycyrrhetinate }\end{array}$ & $\begin{array}{c}\text { Antifungal } \\
\text { Anti-inflammatory, } \\
\text { antioxidant, and skin- } \\
\text { soothing }\end{array}$ \\
$\begin{array}{ll}\text { Dihydroavenanthramide } & \text { Anti-itch, soothing, } \\
& \text { antioxidant, and anti- } \\
& \text { inflammatory } \\
\text { Zinc pidolate } & \text { Sebum-regulating, astringent } \\
\text { Acetamide MEA } & \text { Conditioning agent, } \\
& \text { humectant } \\
\text { Biosaccharide gum-2 } & \text { Anti-inflammatory, soothing } \\
\text { Pentylene } & \text { Anti-irritant, anti-itch, } \\
\text { glycol }+ \text { butylene } \\
\text { glycol }+ \text { hydroxyphenyl } \\
\text { propamidobenzoic acid }\end{array}$ & antihistaminic \\
Polymethyl methacrylate & $\begin{array}{c}\text { Hydrator and moisturization } \\
\text { enhancer }\end{array}$ \\
\hline
\end{tabular}


ingredients, on the other hand, are substances used to help spread or preserve the product such as acetamide monoethanolamine (MEA), hyaluronic acid (HA), and polymethyl methacrylate (PMMA). They affect the look and feel of the product and they are also referred to as the vehicle (Fig. 1).

\section{Performance Ingredients}

\section{Piroctone Olamine}

Piroctone olamine is a hydroxamic acid that inhibits ergosterol synthesis, the main component of the cell wall of most fungi. It is used as a cream or shampoo at a $0.5-1 \%$ concentration. Piroctone olamine alone or in combination with other agents has been demonstrated to reduce erythema, scaling, burning/stinging sensation, and pruritus in patients with FSD, with excellent cosmetic results [17-20].

\section{Stearyl Glycyrrhetinate}

Stearyl glycyrrhetinate (SG) is a fatty acid with a highly oil-soluble structure that easily dissolves in several types of lipids. This high lipophilicity allows SG an excellent ingredient for the oily skin of FSD patients. Stearyl glycyrrhetinate has skin-soothing and mattifying properties. Its tolerability and cosmetic qualities allows SG a good option to consider when dealing with mild-to-moderate SD [21].

\section{Avenanthramides}

Avenanthramides are a heterogenous group of compounds with antihistaminic and anti-inflammatory properties. This allows them suitable for use in a variety of skin conditions that cause dryness, eczema, irritation, and inflammation, as occurs in FSD. Clinical studies have demonstrated that topical use significantly alleviated itching and improved the condition of moderately to severely dry skin, and was well tolerated patients with itching [22-24].

\section{Zinc PCA}

The zinc salt of L-pyrrolidone carboxylate (zinc PCA) has long been used for its astringent and antifungal properties. It also has antioxidative and anti-inflammatory effects. Zinc PCA is a good option for patients with mild exudative FSD [25-27].
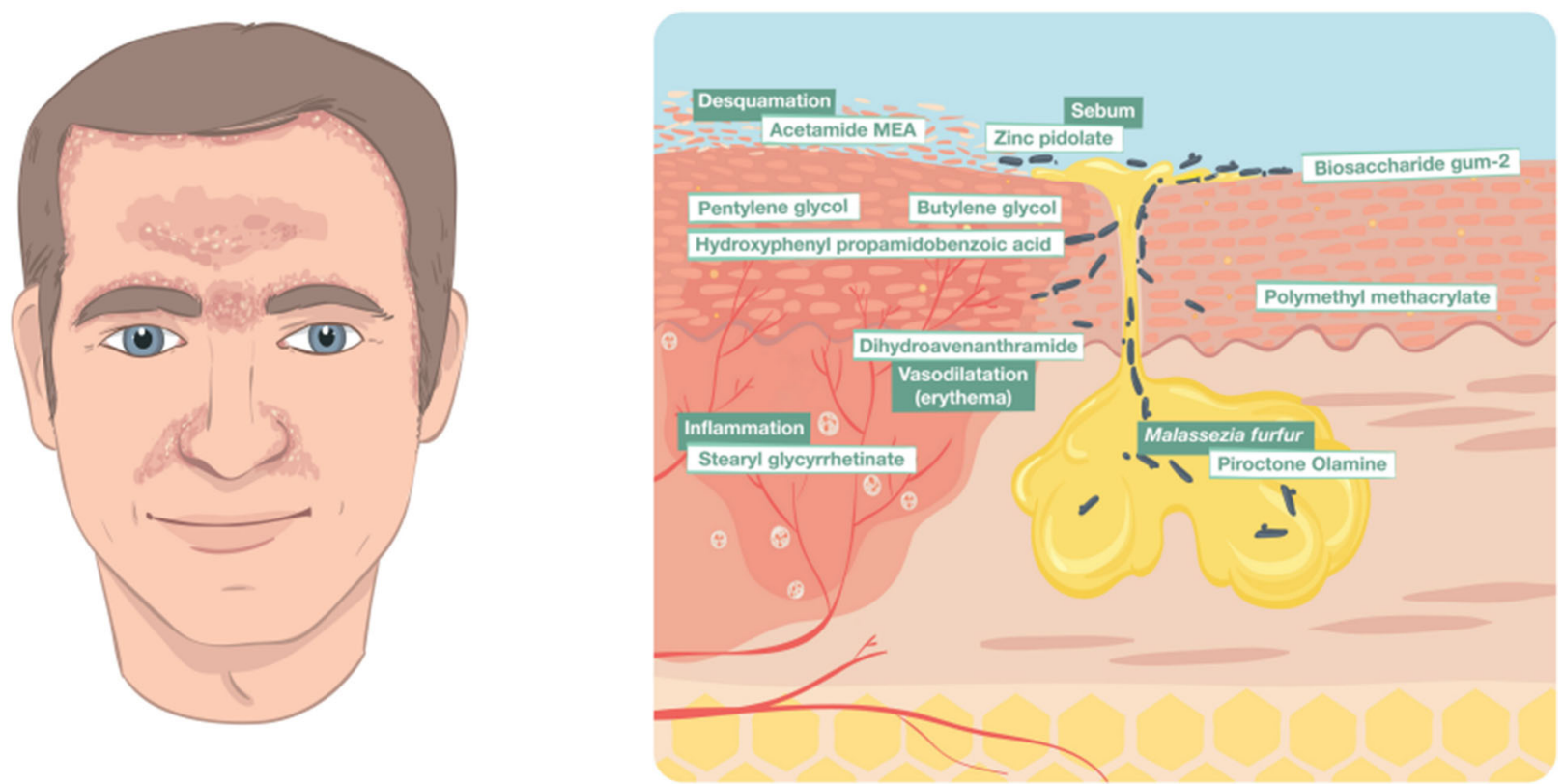

Fig. 1 Seborrheic dermatitis following the typical distribution on the face. The scheme presents the cross section of the skin structure and the specific properties of the non-pharmacological anti-FSD ingredients 


\section{Biosaccharide Gum-2}

Biosaccharide gum-2 has anti-inflammatory and soothing active properties, protecting the skin against external aggressions. Biosaccharide gum-2 cream, in combination with zinc PCA, piroctone olamine, dihydroavenanthramide, and stearyl glycyrrhetinate, has been demonstrated to be effective in the treatment of mild FSD [28].

\section{Functional Ingredients}

Optimal hydration of the surface of the skin protects the underlying cutaneous tissues and maintains normal processes of keratinocyte proliferation and epidermal differentiation [13].

Moisture plays an important role in skin protection and normal epidermal differentiation. Therefore, keeping the facial skin moisturized helps to protect the skin from environmental insults that could result in inflammation and symptoms such as erythema, desquamation, and pruritus $[2,5,13]$. Patients with FSD have seborrheic skin, so topical products should be in the form of an oil in water light cream. FSD involves an imbalance of skin lipids with a higher concentration of triglycerides and cholesterol and lower concentrations of squalene. Release of free fatty acids from triglycerides irritates the skin, inducing erythema, itchiness, and desquamation. The use of inappropriate creams may therefore induce additional irritation. Light creams, emulsions, and gels provide the best vehicle, as they do not worsen the symptoms of FSD.

\section{Acetamide Monoethanolamine (MEA)}

MEA is the International Nomenclature of Cosmetic Ingredients (INCI) name of the 75\% aqueous solution of the monoethanolamide of acetic acid. Functionally, it may be classified as a humectant, an antistatic agent, a plasticizer, or a lubricant. Its use in skincare and haircare is facilitated by its $\mathrm{pH}$ stability range and compatibility with anionic, cationic, and nonionic surfactants [19]. It functions to increase skin moisture content in the upper layers by drawing moisture from the environment.

\section{Polymethyl Methacrylate (PMMA)}

PMMA offers hydration acting as a lubrication enhancer in cosmetics. In its purified form, topically, helps fill wrinkles and provides a smoother visible surface $[17,28]$.

\section{Hyaluronic Acid}

Hyaluronic acid (HA) is a complex carbohydrate polymer that has an important role in skin hydration and cell-to-cell communications. As a topical formulation, $\mathrm{HA}$ is a well-known enhancer of topical delivery drugs. Topical application of HA sodium salt gel $0.2 \%$ and $5 \%$ cream has been shown to reduce the signs and symptoms of FSD. The beneficial effects of HA seem to be exerted by inducing the epidermal innate immune system against local aggressive microorganisms [29].

\section{Sunscreens for FSD}

Patients with FSD are more sensitive to ultraviolet, infrared, and visible light radiation and therefore need sunscreens that protect against a wide-ranging electromagnetic spectrum $[6,7]$. In addition, several factors including chemicals, preservatives, and the vehicle of the product can affect patients' skin $[4,5,13]$.

HA is a polysaccharide that occurs naturally in the healthy dermis. Sunscreens with HA sodium salts have been demonstrated to improve all symptoms of FSD [29].

Other polymers such as PMMA can also be useful, as they improve extensibility and adhesion to the skin and absorb sweat and sebum, which helps to eliminate sheen from the skin and from other ingredients, thus improving the cosmetic acceptability of topical treatments $[17,28]$.

In general, the ideal sunscreen for patients should have a high SPF for UVB and UVA, be non-irritating and non-comedogenic, with a matte appearance, immediate absorption, and contain HA and particles of polymers in a waterbased (non-oily) formula. 


\section{Cleansers for FSD}

Skin cleansing-removal of liposoluble, hydrosoluble, and insoluble dirt-is essential in the care of both disease-affected and disease-free skin $[18,22]$. The numerous cleansing products available have various different mechanisms of action, and include surfactants, makeup removers, astringents, and abrasive cleansers $[2,18]$. It is important to take into account the quantity of sebum and degree of irritation when choosing the most suitable cleanser. Some patients may have a thick, seborrheic cutis and require gels and shampoos, while other patients may have a dry, sensitive, rosaceiform skin and require foam, micellar water, and lipid-free products $[4,17]$.

\section{Sebum-Controlling Agents}

FSD patients often have overproduction of sebum, driven by androgenic stimulation of the sebaceous glands [9]. Topical sebum-regulating agents can reduce skin oiliness and are available in different formulations such as emulsions, gels, or lotions. Their action is generally attributed to mattifying agents such as methacrylate copolymer microspheres that absorb sebum from the skin surface $[17,28]$.

\section{Antimicrobial Agents}

Cosmetics containing antimicrobial agents offer a potential treatment option in patients with mild inflammatory FSD on long-term topical steroids, though they may also be suitable when patients are taking maintenance therapy or have discontinued pharmacological treatment. Since they are non-photosensitizing, their use their use in summertime does not need to be restricted. Patients with mild or moderate FSD may also benefit from topical antimicrobials as an adjunct to standard pharmacological treatments without need for modification of the existing treatment or patient behavior $[8,10,13]$.

\section{Anti-Inflammatory Agents}

Recent advances in understanding the pathogenesis of FSD have suggested a pivotal role of inflammatory processes in the development of SD lesions [4]. Most topical and systemic pharmacological agents for SD exert their effect via anti-inflammatory actions, and it therefore seems reasonable to use cosmetics that also possess anti-inflammatory properties [13].

\section{Skincare Routine for FSD Patients}

Facial seborrheic dermatitis is a chronic disease with most of the patients showing periods of remissions and flares. It is therefore important for patients to have a daily basic skincare routine during remissions and a proactive treatment during the flares. We recommend facial cleansing with gentle soaps twice a day. In the morning start with syndet cleanser foam to clean the face followed by the application of a non-pharmacological gel or cream on all the face including the ears. Choosing a gel or a cream will depend on the patient's skin type, the gel being more appropriate in patients with greasy skin. Afterwards a high-SPF sunscreen

Table 3 Non-pharmacological topical routine for FSD: recommendations for patients

In the morning

1. Start with a gentle syndet foam cleanser

2. Apply a specific gel/cream to the whole face, including the ears

3. Use an o/w SPF $50+$ sunscreen with or without color

At night

1. Micellar water to remove cosmetics or makeup

2. Syndet cleanser

3. Non-pharmacological topical gel/cream

4. In case of flare-ups apply topical drug treatment only to areas with clinical disease 
containing HA or PMMA in a water-based (nonoily) formula is recommended. If desired a light layer of a non-comedogenic and high-tolerance makeup can be applied. At night remove the makeup with micellar water and clean the whole face with syndet cleanser foam and apply the non-pharmacological gel or cream. During the flares patients must follow the same cleaning routine as in the remission periods. If topical treatment is indicated, this should be applied directly to the affected skin after the cleaning. The non-pharmacological gel or cream can be applied afterwards (Table 3).

\section{CONCLUSIONS}

Facial seborrheic dermatitis is a common dermatological disorder worldwide, with a high incidence during puberty and a peak in the third and fourth decades of life. Multiple factors have been identified as predisposing to FSD, and understanding of the causes and pathogenesis of FSD has improved thanks to advances in microbiological and analytical techniques. The key factors of proliferation of Malassezia yeast, the state of the epidermis, and high levels of sebum secretion interact, along with other factors, to potentially culminate in FSD.

The goal of treatment should be to provide safe and effective treatment that improves symptoms, reduces recurrence, and causes minimal side effects. As the nature of the condition is chronic, treatment should aim to control the frequency and severity of flare-ups. Managing FSD often requires drug treatments in the form of topical antifungals, keratolytics, corticosteroids, and calcineurin inhibitors.

Appropriate prescription and use of cosmetic products and procedures can improve treatment outcomes, while their inappropriate use can worsen FSD. The choice of treatments should be based on the severity of the condition as well as any existing pharmacological treatments. Establishing recommendations on non-pharmacological treatments that identify the indications and appropriate precautions could be helpful for clinicians in deciding the best option for their patients.
A multi-targeted therapy facial cream that has anti-inflammatory, antifungal, sebum-controlling, and anti-scaling properties is ideal. This topical, steroid-free, fragrance-free, and waterbased emulsion for the management of the signs and symptoms of seborrhea and seborrheic dermatitis could be useful for daily routine use, controlling the symptoms without use of pharmacological therapy.

\section{ACKNOWLEDGEMENTS}

Funding. No funding or sponsorship was received for this study or publication of this article. The Rapid Service Fee was funded by the authors. The authors are fully responsible for all content and received no financial support or any other form of compensation related to the development of this manuscript.

Authorship. All named authors meet the International Committee of Medical Journal Editors (ICMJE) criteria for authorship for this article, take responsibility for the integrity of the work as a whole, and have given their approval for this version to be published.

Medical Writing and/or Editorial Assistance. Special thanks to Dr. Jane Marshall (freelance) for the medical writing assistance. Support for this assistance was funded by the authors.

Disclosures. Dr. Jaime Piquero-Casals is consultant of ISDIN. Doris Hexsel, Juan Francisco Mir-Bonafé, and Eduardo Rozas-Muñoz have nothing to disclose.

Compliance with Ethics Guidelines. This article is based on previously conducted studies and does not contain any studies with human participants or animals performed by any of the authors.

Data Availability. Data sharing is not applicable to this article as no datasets were generated or analyzed during the current study. 
Open Access. This article is distributed under the terms of the Creative Commons Attribution-NonCommercial 4.0 International License (http://creativecommons.org/licenses/ by-nc/4.0/), which permits any noncommercial use, distribution, and reproduction in any medium, provided you give appropriate credit to the original author(s) and the source, provide a link to the Creative Commons license, and indicate if changes were made.

\section{REFERENCES}

1. Sampaio AL, Mameri AC, Vargas TJ, Ramos-e-Silva M, Nunes AP, Carneiro SC. Seborrheic dermatitis. An Bras Dermatol. 2011;86(6):1061-71 (Quiz 1072-4).

2. Gupta AK, Madzia SE, Batra R. Etiology and management of seborrheic dermatitis. Dermatology. 2004;208(2):89-93.

3. Naldi L. Seborrhoeic dermatitis. BMJ Clin Evid. 2010;2010:1713.

4. Borda L, Wikramanayake T. Seborrheic dermatitis and dandruff: a comprehensive review. J Clin Investig Dermatol. 2015;3(2). https://doi.org/10. 13188/2373-1044.1000019.

5. Bukvic ZM, Kralj M, Basta-Juzbasic A, Lakos J. Seborrhoeic dermatitis: an update. Acta Dermatovenerol Croat. 2012;20(2):98-104.

6. Sanders MGH, Pardo LM, Franco OH, Ginger RS, Nijsten T. Prevalence and determinants of seborrhoeic dermatitis in a middle-aged and elderly population: the Rotterdam study. Br J Dermatol. 2018;178(1):148-53.

7. Maietta G, Rongioletti F, Rebora A. Seborrheic dermatitis and daylight. Acta Derm Venereol. $1991 ; 71(6): 538-9$.

8. Faergemann J, Borgers M, Degreef H. A new ketoconazole topical gel formulation in seborrhoeic dermatitis: an updated review of the mechanism. Expert Opin Pharmacother. 2007;8(9):1365-71.

9. Thiboutot D, Jabara S, McAllister JM, et al. Human skin is a steroidogenic tissue: steroidogenic enzymes and cofactors are expressed in epidermis, normal sebocytes, and an immortalized sebocyte cell line (SEB-1). J Investig Dermatol. 2003;120:905-14.
10. Hay RJ, Graham-Brown RA. Dandruff and seborrhoeic dermatitis: causes and management. Clin Exp Dermatol. 1997;22:3-6.

11. Dawson TL. Malassezia globosa and restricta: breakthrough understanding of the etiology and treatment of dandruff and seborrheic dermatitis through whole-genome analysis. J Investig Dermatol Symp Proc. 2007;12(2):15-9.

12. Karakadze MA, Hirt PA, Wikramanayake TC. The genetic basis of seborrhoeic dermatitis: a review. J Eur Acad Dermatol Venereol. 2018;32(4):529-36.

13. Gupta A, Versteeg S. Topical treatment of facial seborrheic dermatitis: a systematic review. Am J Clin Dermatol. 2017;18(2):193-213.

14. Korting HC, Schöllmann C. Medical devices in dermatology: topical semi-solid formulations for the treatment of skin diseases. J Dtsch Dermatol Ges. 2012;10(2):103-9.

15. Draelos ZD. Cosmetic dermatology: products and procedures. New York: Willey-Blackwell; 2010. p. 532. ISBN 978-1-405-18635-3.

16. Newburger AE. Cosmeceuticals: myths and misconceptions. Clin Dermatol. 2009;27(5):446-52.

17. Dall'Oglio F, Tedeschi A, Fusto CM, Lacarrubba F, Dinotta F, Micali G. A novel cosmetic antifungal/ anti-inflammatory topical gel for the treatment of mild to moderate seborrheic dermatitis of the face: an open-label trial utilizing clinical evaluation and erythema-directed digital photography. G Ital Dermatol Venereol. 2017;152:436-40.

18. Schmidt-Rose T, Braren S, Fölster H, et al. Efficacy of a piroctone olamine/climbazol shampoo in comparison with a zinc pyrithione shampoo in subjects with moderate to severe dandruff. Int J Cosmet Sci. 2011;33(3):276-82.

19. Granger C, Goñi F, Martinez G, Garre A. Ex-vivo determination of antifungal activity of a new prescription non-steroidal facial cream against Malassezia furfur in human skin explants. SKIN J Cutan Med. 2017;1:137.

20. Okokon E, Verbeek J, Ruotsalainen J, Ojo O, Bakhoya V. Topical antifungals for seborrhoeic dermatitis. Cochrane Database Syst Rev. 2015;4:CD008138.

21. Oyama K, Kawada-Matsuo M, Oogai Y, Hayashi T, Nakamura N, Komatsuzawa H. Antibacterial effects of glycyrrhetinic acid and its derivatives on Staphylococcus aureus. PLoS One. 2016;11(11):e0165831. 
22. Puglia C, Rizza L, Offerta A, Gasparri F, Giannini V, Bonina F. Formulation strategies to modulate the topical delivery of anti-inflammatory compounds. J Cosmet Sci. 2013;64(5):341-53.

23. Yang $\mathrm{J}, \mathrm{Ou} \mathrm{B}$, Wise $\mathrm{ML}, \mathrm{Chu} \mathrm{Y}$. In vitro total antioxidant capacity and anti-inflammatory activity of three common oat-derived avenanthramides. Food Chem. 2014;1(160):338-45.

24. Jeong-Mi K, Eun-Mi N, Kang-Beom K, et al. Dihydroavenanthramide D prevents UV-irradiated generation of reactive oxygen species and expression of matrix metalloproteinase- 1 and -3 in human dermal fibroblasts. Exp Dermatol. 2013;22(11):759-61.

25. Takino Y, Okura F, Kitazawa M, Iwasaki K, Tagami H. Zinc L-pyrrolidone carboxylate inhibits the UVAinduced production of matrix metalloproteinase-1 by in vitro cultured skin fibroblasts, whereas it enhances their collagen synthesis. Int J Cosmet Sci. 2012;34(1):23-8.
26. Rostan EF, DeBuys HV, Madey DL, et al. Evidence supporting zinc as an important antioxidant for skin. Int J Dermatol. 2002;41:606-11.

27. Lang C, Murgia C, Leong M, et al. Antiinflammatory effects of zinc and alterations in zinc transporter mRNA in mouse models of allergic inflammation. Am J Physiol Lung Cell Mol Physiol. 2007;292:L577-84.

28. Valdera-Martinez P, Garre A, Granger C. Efficacy and tolerance of a new non-steroidal prescription cream in the treatment of mild facial seborrheic dermatitis. SKIN J Cutan Med. 2017;1:s134.

29. Schlesinger T, Powell C. Efficacy and safety of a low molecular weight hyaluronic acid topical gel in the treatment of facial seborrheic dermatitis final report. J Clin Aesthet Dermatol. 2014;7(5):15-8. 